\title{
Integração de um mecanismo de Mineração de Dados Educacionais ao Moodle
}

\author{
Leandro C. Santana ${ }^{1}$, Alexandre M. Maciel${ }^{1}$, Rodrigo L. Rodrigues ${ }^{2}$ \\ ${ }^{1}$ Universidade de Pernambuco (UPE) \\ Programa de Pós-Graduação em Engenharia da Computação (PPGEC) \\ ${ }^{2}$ Universidade Federal Rural de Pernambuco (UFRPE) \\ Departamento de Educação (DEd) \\ \{lsc, amam\}@ecomp.poli.br, rlreded.ufrpe.br
}

\begin{abstract}
Virtual Learning Environments (VLE) are an example of new systems being developed as part of educational environments. Moodle is an example of a VLE distribution which offers courses and training to several persons. In 2012 the Moodle plugin "Avatar Educação" was developed and with it is possible to create personalized notifications and messages within Moodle. This paper proposes an integration mechanism for the "Avatar Educação", based on decision trees, which offers a better control and distribution of data within the plugin. This way, we provide a better interactivity between the users and the VLE.
\end{abstract}

Resumo. Um ambiente educacional a cada dia vem incorporando software em suas atividades, os Ambientes Virtuais de Aprendizagem (AVA) são exemplos desse software. $O A V A$, é uma forma de oferecer cursos e treinamentos e tem como uma das suas distribuições, o Moodle. Em 2012 surgiu o "Avatar Educação", um plugin desenvolvido para notificações e mensagens personalizadas integrada ao Moodle. Este trabalho objetiva a integração de um mecanismo baseado em árvore de decisão integrado ao Avatar Educação afim de oferecer respostas inteligentes a comunidade de Ensino a Distância. Desta forma é possível obter uma interatividade melhor entre os usuários e o ambiente.

\section{Introdução}

O processo de ensino, seja presencial ou ensino a distância (EAD), leva em consideração a interação entre os atores envolvidos no processo: alunos, professores e ambiente. A interação entre esses atores se torna importante dentro do processo, pois proporcionam maior entendimento na relação entre professores e alunos, o acompanhamento é de forma cooperativa e a rede de ensino é influenciada positivamente (SILVA, 2001).

$\mathrm{Na}$ pesquisa de Maciel (2014), intitulada, Avatar Educação, deu início a um processo de melhoria de interação entre os atores envolvidos no processo educacional, porém apresentou limitações de funcionalidades. As consultas realizadas pelo Avatar ao banco de dados foram desenvolvidas de forma estática e não agregam nenhum 
mecanismo inteligente na busca por informação. Isto acarreta um desinteresse gradativo dos alunos e em longo prazo deixam de contribuir para o incremento na interatividade do ambiente (Romero et al., 2008).

Diante desta problemática, este trabalho tem como objetivo apresentar a integração de um mecanismo de mineração de dados educacionais ao ambiente educacional Moodle, a fim de melhorar a interação com os usuários do ambiente.

\section{Mineração de Dados Educacionais}

Romero, Ventura e García (2008) afirmam que na EAD a quantidade de armazenamento de dados vem crescendo, e isso se dá ao fato que as interações como acessos a fóruns, perguntas e respostas na área de questionários e comunicação entre participantes. Diante dessas atividades um grande volume de dados é desenvolvido dificultando uma análise de forma manual.

As técnicas de mineração de dados foram desenvolvidas a muitos anos para ser aplicado em áreas de marketing, vendas e apoio financeiros (Fayyad et al., 1996; Witten et al., 2011) mais recentemente, vem sendo aplicada na área educacional (Baker, 2011). $\mathrm{Na}$ área de educação, Zorrilla et al. (2005) apontam que estas técnicas podem ser aplicadas para diferentes atores que são: professores, estudantes e os gestores. Onde cada um tem sua característica e possui seu ponto de vista.

Diversas abordagens e algoritmos são utilizados na mineração de dados: regressão linear, redes neurais, máquinas de vetores de suporte e árvores de decisão. Romero et al. (2008) salientam que dentre os diversos métodos de mineração, destacam-se as árvores de decisão e as redes neurais, onde as árvores de decisão abordam um conjunto de dados e uma classificação seguindo um caminho que satisfaça as condições, onde inicia-se pela raiz até a folha da árvore. Abordaremos mais especificamente as técnicas de árvore de decisão foco deste trabalho, tendo em vista que a técnica tem melhor resultado com base em experimentos em base de dados educacionais (GOTTARDO, 2014).

\section{1. Árvore de Decisão}

Árvore de decisão é uma técnica de aprendizado de máquina que aborda uma estrutura de árvore para tomada de decisão. A árvore possui vários nós, onde cada nó corresponde a uma tomada de decisão baseada por um determinado dado de entrada. A ligação entre um nó e outro é chamado de ramo, e o último nó no qual não tem mais ligação é chamado de folha, que é o retorno da árvore (QUINLAN, 1993).

A Figura 1 ilustra a estrutura de uma árvore de decisão onde cada nó é realizado uma decisão, e ao chegar na folha, é retornada uma resposta para a mineração. No caso proposto deste trabalho, a resposta da árvore são as opções da classe de entrada no classificador. 


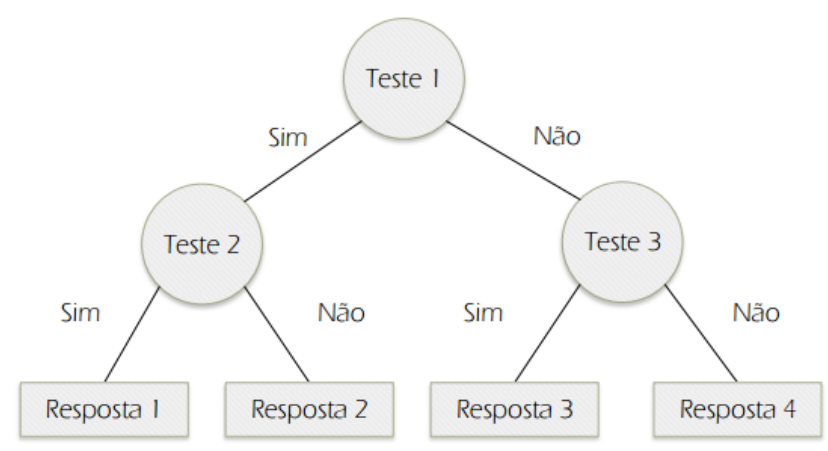

Figura 1. Estrutura de uma árvore de decisão (Rolim, 2014).

Árvore de decisão tem sido largamente utilizada nos trabalhos de mineração de dados educacionais (WANG, 2009). Por este motivo exploramos dos dois principais algoritmos que envolvem os estudos com árvore de decisão, são eles C4.5 (J48) e Random Forest.

\section{Experimentos e Resultados}

Os experimentos deste trabalho foram desenvolvidos em dois cenários: o primeiro foi uma experiência com 79 estudantes e com 6 atributos de uma base de dados do Moodle e foi relatada em Cavalcanti et al. (2014). O experimento do segundo cenário foi realizado com uma base de dados ampla e vários cursos e disciplinas com 1254 alunos e 19 atributos. A característica dos atributos pode ser vista na Teoria da Interação proposta por Moore (1989), onde o mesmo define os dados em dimensões. Neste trabalho as dimensões são chamadas da seguinte forma: Perfil de Uso do AVA, Interação Estudante - Estudante e Interação Bidirecional. A relação Dimensão $\mathrm{x}$ Atributos é dada a seguir:

- Perfil de Uso do AVA

$\checkmark$ Número total de acesso ao AVA;

$\checkmark$ Número de postagens de outros participantes lidas em fóruns;

$\checkmark$ Número total de revisões em postagens anteriores realizadas em fóruns;

$\checkmark$ Número de sessões de chat que o estudante participou;

$\checkmark$ Número de mensagens enviadas ao chat;

$\checkmark$ Número de questões respondidas;

$\checkmark$ Número de questões respondidas corretamente;

$\checkmark$ Tempo médio decorrido entre os diversos acessos ao sistema;

$\checkmark$ Número de dias transcorridos entre o início do curso e o primeiro acesso do estudante no AVA.

- Interação Estudante - Estudante

$\checkmark \quad$ Número total de respostas postadas em fóruns referindo-se a postagens de outros estudantes; 
VI Congresso Brasileiro de Informática na Educação (CBIE 2017)

Anais dos Workshops do VI Congresso Brasileiro de Informática na Educação (WCBIE 2017)

$\checkmark \quad$ Número de postagens em fóruns de outros estudantes que fazem referência a postagem do estudante;

$\checkmark$ Número de mensagens recebidas de outros estudantes durante a realização do curso;

$\checkmark \quad$ Número de mensagens enviadas a outros estudantes durante a realização do curso.

- Interação Bidirecional

$\checkmark \quad$ Número de postagens do estudante que tiveram respostas feitas por professores ou tutores do curso;

$\checkmark \quad$ Número de postagens de professores ou tutores que tiveram respostas feitas pelo estudante;

$\checkmark \quad$ Número de mensagens enviadas ao professor/tutor durante a realização do curso;

$\checkmark$ Número de mensagens recebidas do professor/tutor durante a realização do curso.

O objetivo da previsão é o resultado final obtido pelo estudante no curso. Representa a classe objetivo de classificação. Nesse experimento foi utilizado técnicas de mineração Randon Forest e J48 por meio da ferramenta Weka. Na Tabela 1 é possível visualizar a taxa de acerto usando as técnicas.

\begin{tabular}{|c|c|}
\hline $\mathbf{R F}$ & $\mathbf{J 4 8}$ \\
\hline $94,37 \%$ & $94,69 \%$ \\
\hline
\end{tabular}

Tabela 1. Acurácia dos Algoritmos

Diante dos resultados, chegamos a uma performance de $94,69 \%$ do algoritmo J48. Para ilustrar melhor o resultado, a Figura 2 e 3 mostram os resultados e matriz de confusão do experimento.

$\begin{array}{lcr}\text { Correctly Classified Instances } & 1195 & 94.691 \\ \text { Incorrectly Classified Instances } & 67 & 5.309 \\ \text { Kappa statistic } & 0.6927 & \\ \text { Mean absolute error } & 0.0664 & \\ \text { Root mean squared error } & 0.2195 & \\ \text { Relative absolute error } & 37.6181 \% \\ \text { Root relative squared error } & 74.0038 \% & \\ \text { Total Number of Instances } & 1262 & \end{array}$

Figura 2. Resultado do Algoritmo J48

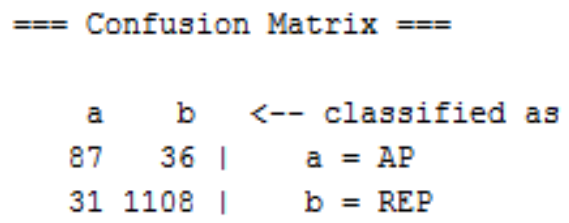

Figura 3. Representa o resultado da Matriz de Confusão j48 
VI Congresso Brasileiro de Informática na Educação (CBIE 2017)

Anais dos Workshops do VI Congresso Brasileiro de Informática na Educação (WCBIE 2017)

Para todos os experimentos dos cenários se utilizou da normalização padrão, minmáx e z-score.

\subsection{Integração ao Moodle}

Após realização dos experimentos e escolha da melhor técnica de mineração em relação a série de dados escolhidas, a integração ao Moodle aconteceu da seguinte maneira:

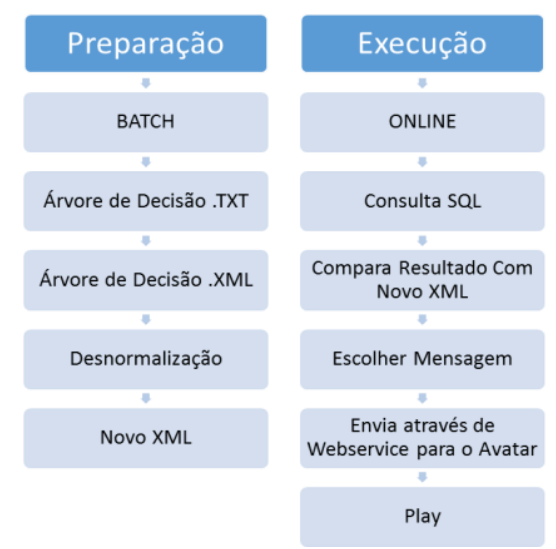

Figura 4. Fases de desenvolvimento do mecanismo de inteligência

A Figura 4 caracteriza a etapa de desenvolvimento em dois cenários distintos, onde inicia-se com a preparação dos dados, em seguida a fase de execução. A preparação dos dados foi executada localmente (localhost), onde foi criado o arquivo .TXT contendo a árvore de decisão gerado pela saída do Weka. O arquivo .TXT foi importado ao plugin WekaTextToXML e gerado um .XML logo em seguida. Após o arquivo XML ter sido gerado, o próximo passo foi de desnormalizar os dados, tendo em vista que os dados foram normalizados inicialmente para poder ser aplicado as técnicas de mineração. Após essa desnormalização, um novo arquivo XML foi gerado.

O cenário da execução dos dados se dá por meio do servidor online do Moodle, onde o aluno faz o login com seu ID e o Avatar Educação faz consultas na base de dados do aluno, fazendo comparações ao novo XML (árvore de decisão) escolhendo a mensagem que caracteriza o perfil do aluno e executando o áudio da mensagem por meio da voz sintetizada.

As mensagens foram divididas em duas categorias, mensagens motivadoras e mensagens de alerta como pode ser vista na Tabela 2 e na Tabela 3. Ambas transmitem estímulos para o estudante seguir motivado no curso. As mensagens motivadoras, são selecionadas quando a folha da árvore tem como resultado "aprovado", já as mensagens de alerta, tem uma propriedade de motivar o aluno a melhorar dentro da disciplina ou curso, e estas mensagens são selecionadas quando a folha da árvore tem como resultado "reprovado".

\begin{tabular}{|l|l|}
\hline \multicolumn{2}{|c|}{ MENSAGEM MOTIVADORA } \\
\hline NR_MSG_REC_PROF & $\begin{array}{l}\text { Parabéns pela comunicação com seu tutor! Continue } \\
\text { assim! }\end{array}$ \\
\hline NR_QUESTOES_RESP & $\begin{array}{l}\text { Continue fazendo os exercícios! Isso é ótimo para } \\
\text { ajudar no seu bom desempenho! }\end{array}$ \\
\hline NR_MSG_CHAT & $\begin{array}{l}\text { Parabéns pelo uso constante do chat. Continue assim! } \\
\text { As trocas com tutores e colegas podem ser muito }\end{array}$ \\
\hline
\end{tabular}


VI Congresso Brasileiro de Informática na Educação (CBIE 2017)

Anais dos Workshops do VI Congresso Brasileiro de Informática na Educação (WCBIE 2017)

\begin{tabular}{|c|c|}
\hline & enriquecedoras. \\
\hline RESP_PROF_EST & $\begin{array}{l}\text { Parabéns por sua interação no fórum! Continue } \\
\text { comunicando-se com seu professor, tutor e colegas! }\end{array}$ \\
\hline TEMPO_DECORRIDO & $\begin{array}{l}\text { Você vem acessando o ambiente regularmente. Isso vai } \\
\text { ajudar no seu rendimento! }\end{array}$ \\
\hline TEMPO_DECOR_PRIM_LOGIN & $\begin{array}{l}\text { Sua frequência de acesso no ambiente está muito boa! } \\
\text { Continue sempre assim! }\end{array}$ \\
\hline NR_POSTS_REC & $\begin{array}{l}\text { Continue sempre acessando os fóruns, compartilhando } \\
\text { experiências com outros alunos! }\end{array}$ \\
\hline NR_TOT_POST & $\begin{array}{l}\text { Continue sempre interagindo no fórum! Sua participação } \\
\text { está ótima! }\end{array}$ \\
\hline NR_QUESTOES_ACERT & $\begin{array}{l}\text { Continue respondendo sempre as atividades! Ótimo } \\
\text { trabalho! }\end{array}$ \\
\hline NR_MSG_ENV_EST_PROF & $\begin{array}{l}\text { Percebo que você sempre busca seu tutor para tirar as } \\
\text { dúvidas! Isso é ótimo! Continue assim! }\end{array}$ \\
\hline NR_MSG_REC_EST & $\begin{array}{l}\text { Procure sempre ajudar seus colegas no que } \\
\text { precisarem. }\end{array}$ \\
\hline NR_LOGINS & $\begin{array}{l}\text { Continue sempre acessando o ambiente. Muito bom } \\
\text { isso. }\end{array}$ \\
\hline NR_CHATS & $\begin{array}{l}\text { Você está tendo uma ótima comunicação com os } \\
\text { participantes, muito bem. }\end{array}$ \\
\hline
\end{tabular}

Tabela 2. Mensagens motivadoras geradas pelo Avatar

\begin{tabular}{|l|l|}
\hline \multicolumn{2}{|c|}{ MENSAGEM DE ALERTA } \\
\hline RESP_EST_PROF & $\begin{array}{l}\text { Procure verificar sempre os fóruns. Eles são muito } \\
\text { importantes! Bons estudos! }\end{array}$ \\
\hline NR_TOT_POSTS & $\begin{array}{l}\text { Você já interagiu no fórum hoje? Verifique as novidades. } \\
\text { Talvez seu amigo tenha alguma dúvida que você possa } \\
\text { ajudar! }\end{array}$ \\
\hline NR_MSG_ENV_EST & $\begin{array}{l}\text { Lembre-se que os chats são semanais. Aproveite e tire } \\
\text { as dúvidas, se houver! }\end{array}$ \\
\hline NR_TOT_REV & $\begin{array}{l}\text { A interação com o ambiente é muito importante! Nunca } \\
\text { deixe de acessa-lo! }\end{array}$ \\
\hline NR_QUESTOES_ACERT & $\begin{array}{l}\text { Procure reler as anotações de aulas, assistir aos vídeos } \\
\text { e seguir as orientações de estudo do professor. Depois, } \\
\text { tente refazer os exercícios. }\end{array}$ \\
\hline NR_MSG_REC_EST & $\begin{array}{l}\text { Procure ajudar os demais colegas. Verifique se eles } \\
\text { precisam de sua ajuda nos fóruns! }\end{array}$ \\
\hline NR_QUESTOES_RESP & $\begin{array}{l}\text { Não deixe para a última hora. Siga as orientações do } \\
\text { professor e responda o quanto antes os exercícios!! } \\
\text { Bons estudos! }\end{array}$ \\
\hline NR_MSG_REC_PROF & $\begin{array}{l}\text { Você se comunicou com seu tutor recentemente? } \\
\text { Verifique se ele tem novidades para você! }\end{array}$ \\
\hline TEMPO_DECOR_PRIM_LOGIN & $\begin{array}{l}\text { A regularidade de sua participação é essencial para o } \\
\text { sucesso do curso, nunca deixe de acessar a plataforma! }\end{array}$ \\
\hline
\end{tabular}


VI Congresso Brasileiro de Informática na Educação (CBIE 2017)

Anais dos Workshops do VI Congresso Brasileiro de Informática na Educação (WCBIE 2017)

\begin{tabular}{|l|l|}
\hline TEMPO_DECORRIDO & $\begin{array}{l}\text { Sempre procure acessar o ambiente, isso ajudará seu } \\
\text { desempenho nas atividades! }\end{array}$ \\
\hline NR_LOGINS & $\begin{array}{l}\text { Acesse o ambiente regularmente, assim vai melhorar } \\
\text { seu desempenho. }\end{array}$ \\
\hline NR_CHATS & $\begin{array}{l}\text { Converse com seus amigos, veja se eles precisam de } \\
\text { sua ajuda. }\end{array}$ \\
\hline
\end{tabular}

Tabela 3. Mensagens de alerta geradas pelo Avatar

\subsubsection{Moodle/Avatar}

Essa interação dar-se por meio de um personagem animado com voz sintetizada, o Avatar Educação, que pode ser visualizado na Figura 5.

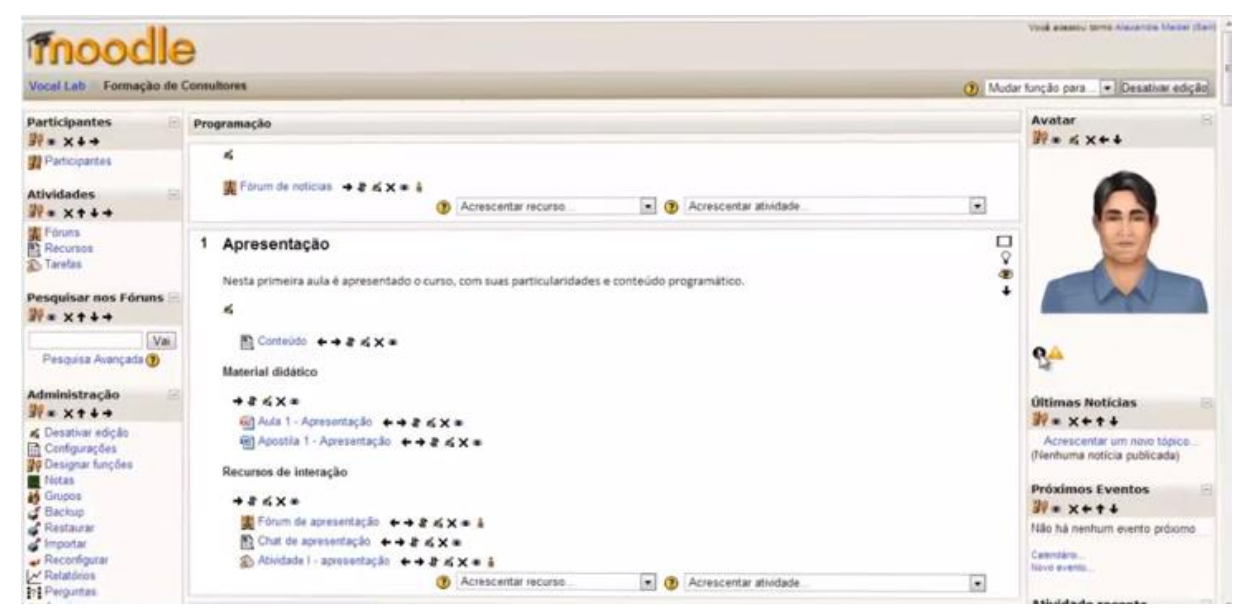

Figura 5. Tela do Ambiente Moodle com o Avatar Educação (Maciel, 2014).

O uso das técnicas de mineração por meio da arvore de decisão favorece o Avatar Educação ao adicionar uma camada mais robusta de processamento de informações subjetivas e contextuais. Na versão anterior, o levantamento de escolha das mensagens a ser enviadas ao usuário se dava baseado em escolhas pré-definidas e que se mostravam não-flexíveis, causando um efeito de mecanização na comunicação. Com a adição da árvore de decisão, conseguimos introduzir flexibilidade e customização individual na comunicação com o usuário, o que tende a aumentar o grau de engajamento do usuário com o sistema.

Essa etapa é a manipulação das classes do plugin Avatar Educação e incluir o mecanismo inteligente em sua estrutura. A estrutura do Avatar Educação antes da inserção do mecanismo de inteligência pode ser vista na Figura 6. 
VI Congresso Brasileiro de Informática na Educação (CBIE 2017)

Anais dos Workshops do VI Congresso Brasileiro de Informática na Educação (WCBIE 2017)

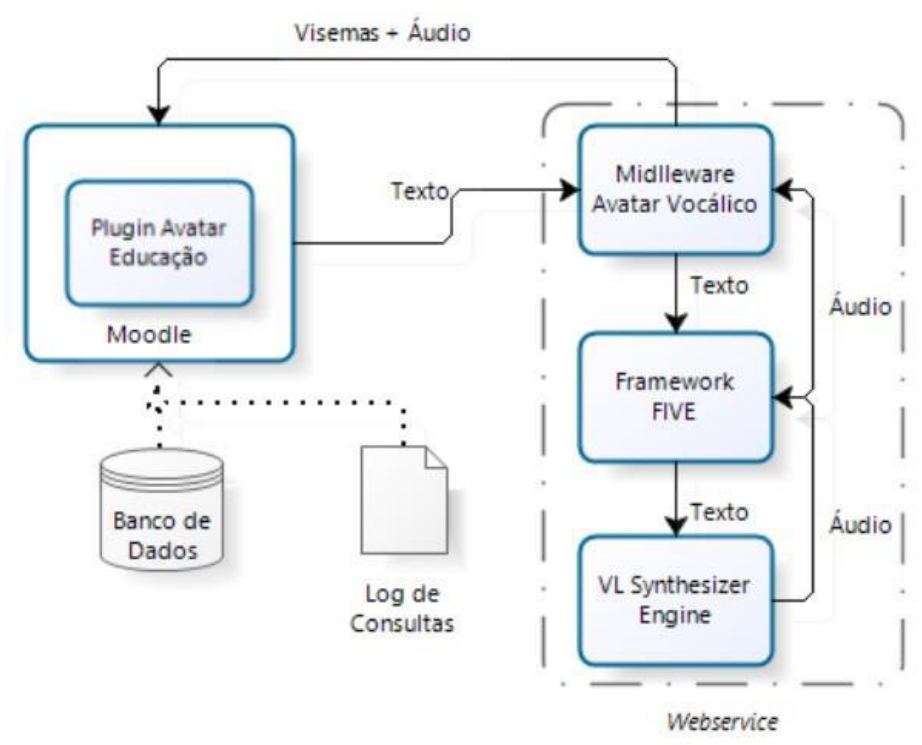

Figura 6. Arquitetura do Avatar sem o mecanismo inteligente

Depois do mecanismo de inteligência ser inserido ao Avatar Educação, a estrutura recebeu uma atualização, que pode ser vista na Figura 7. A mensagem inteligente está diretamente ligada ao mecanismo de mineração, recurso que será inserido ao Avatar Educação para realizar a sintetização da mensagem e reprodução do áudio.

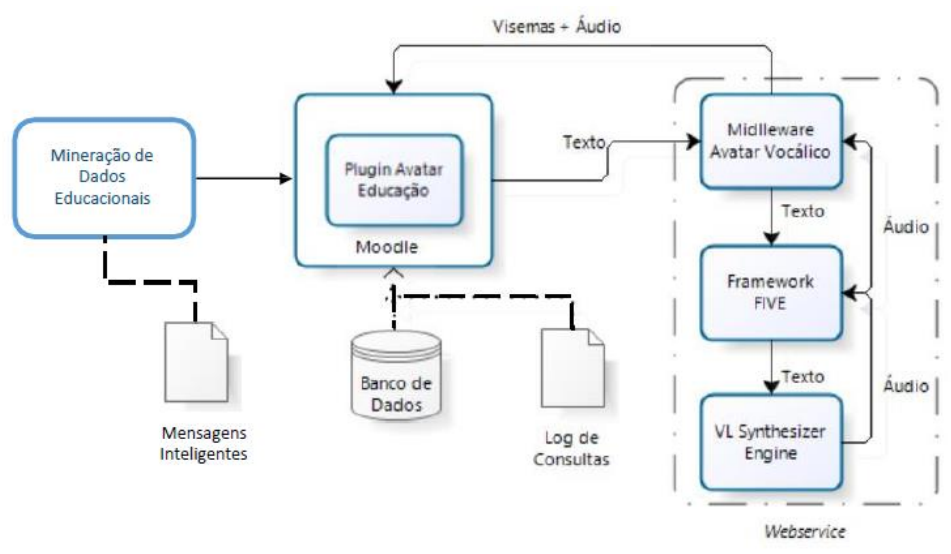

Figura 7. Arquitetura do Avatar com o mecanismo inteligente

Para ilustrar a etapa de desenvolvimento, a Figura 8, mostra as etapas até a execução do mecanismo.
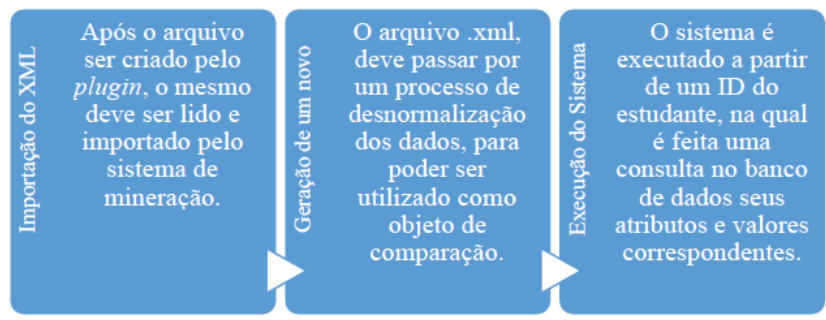

Figura 8. Etapas de execução do desenvolvimento do mecanismo

A Execução do Sistema, inicia-se através do aluno fazendo o login no AVA, ao fazer este procedimento, o Avatar Educação verifica se nas configurações a opção de 
VI Congresso Brasileiro de Informática na Educação (CBIE 2017)

Anais dos Workshops do VI Congresso Brasileiro de Informática na Educação (WCBIE 2017)

mensagens inteligentes está marcada (a configuração pode ser vista na Figura 9). Deste modo, o sistema verifica o ID do aluno e faz comparações dos atributos do aluno logado com os atributos da árvore de decisão, percorrendo a árvore verificando se o aluno foi aprovado ou reprovado.

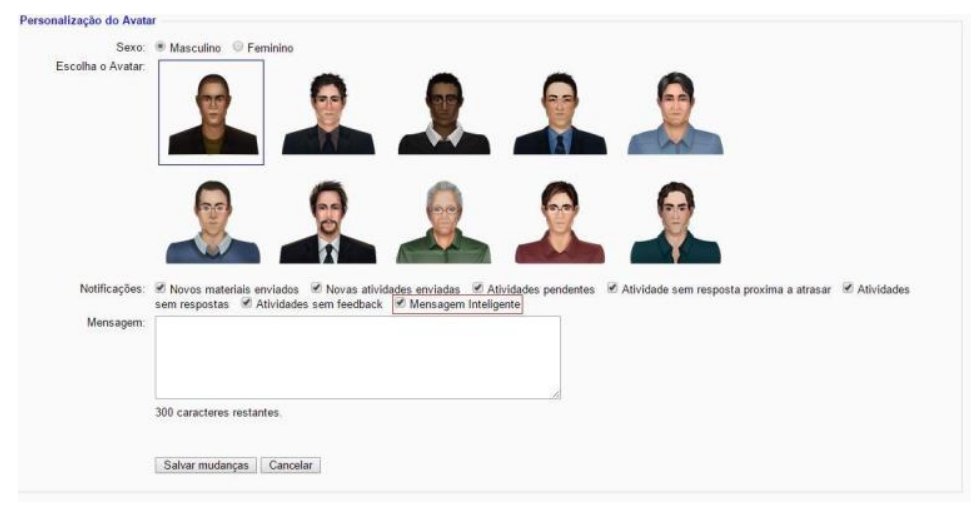

Figura 9. Tela de configuração do Avatar Educação

É importante destacar que o mecanismo de inteligência está voltado para o estudante, ou seja, as mensagens inteligentes e manipulação dos dados é em benefício ao aluno do AVA.

\section{Conclusão}

Este trabalho apresentou um modelo de aplicação na área de mineração de dados educacionais, por meio de um mecanismo ao plugin Avatar Educação. Foi realizado em um ambiente Moodle, um ambiente facilitador de comunicação durante o processo pedagógico, pois oferece uma contribuição para a comunicação entre os professores/tutores e alunos de uma disciplina ou curso.

Analisando-se os melhores resultados dos cenários estudados ao longo dessa pesquisa, percebe-se que as taxas de acerto das técnicas variaram entre $80 \%$ e $94 \%$, podendo ser consideradas expressivas no contexto educacional. Afirmação que se baseia no resultado de Hämäläinen e Vinni (2011) que, por meio de pesquisas relacionadas a desempenho obteve uma taxa média de acerto de $72 \%$ entre as pesquisas.

Do ponto de vista tecnológico é uma nova funcionalidade integrada ao Avatar que torna as mensagens mais dinâmicas, agregando um valor a ferramenta educacional, visando a busca da interatividade maior com os alunos, fazendo com que os mesmos se dediquem em suas atividades no ambiente.

\section{Referências}

Baker, R.S.J.d., I. S. d. C. A. (2011). Mineração de dados educacionais: Jornada de Atualização em Informática na Educação - JAIE 2012 25/29. Oportunidades para o Brasil. Revista Brasileira de Informática na Educação, 19(2).

Cavalcanti, L.; Maciel, A.; Rodrigues, R. (2014). Avaliação do Perfil de Uso no Ambiente Moodle Utilizando Técnicas de Mineração de Dados. Simpósio Brasileiro de Educação a Distância, Dourados, 2014. 
VI Congresso Brasileiro de Informática na Educação (CBIE 2017)

Anais dos Workshops do VI Congresso Brasileiro de Informática na Educação (WCBIE 2017)

Fayyad, U.; Piatetsky-Shapiro, G.; Smyth, P. (1996). Knowledge Discovery and Data Mining: towards a unifying framework. In: Second International Conference ON KD \& DM.

Gottardo, E., Kaestner, C. A. A., Noronha, R. V. (2014). Estimativa de Desempenho Acadêmico de Estudantes: Análise da Aplicação de Técnicas de Mineração de Dados em Cursos a Distância. RBIE, Vol 22, No 1, p. 45-54.

Hämäläinen, W., Vinni, M. (2011). Classifiers for Educational Data Mining. In: Romero et al. Handbook of Educational Data Mining. Flórida, CRC Press, p. 57-71.

Maciel, A. M. A.; Rodrigues, R. L.; Carvalho, E. C. B. (2014). Desenvolvimento de um Assistente Virtual Integrado ao Moodle para Suporte a Aprendizagem Online. Simpósio Brasileiro de Educação a Distância, Dourados.

Moore M. G. (1989). Three Types of Interaction. The American Journal of Distance Education, 3(2):1-6.

Quinlan, J. R. (1993). C4.5: Programs for machine learning. Morgan Kaufmann Publishers Inc., San Francisco, CA, USA.

Romero, C., Ventura, S. e García, E. (2008). Data mining in course management systems: Moodle case study and tutorial. Computers and Education, 51 (1), pp. 368384.

Silva, E., Menezes, E. (2001). Metodologia da Pesquisa e Elaboração de Dissertação. Florianópolis: Universidade Federal de Santa Catarina - UFSC.

Wang, Y. Tseng, M. Liao, H. (2009). Data mining for adaptive learning sequence in English language instruction. Expert Syst. Appl .J.,vol.36, pp. 7681-7686.

Witten, I.H., Frank, E., Hall, M.A. (2011). Data Mining: Practical Machine Learning Tools and Techniques. San Francisco: Morgan Kaufmann, 3 ed.

Zorrilla, M. E., Menasalvas, E., Marin, D., Mora, E., Segovia, J. (2005). Web usage mining project for improving web-based learning sites. In Web Mining Workshop. Cataluna (pp. 1-22).. 449-460. 